\title{
REDES NEURAIS APLICADAS NA INVESTIGAÇÃO DE AVC POR TOMOGRAFIA COMPUTADORIZADA
}

\section{NEURAL NETWORKS APPLIED IN STROKE INVESTIGATION THROUGH COMPUTERIZED TOMOGRAPHY}

\author{
Bruna Kushikawa Silva ${ }^{1}$; Silvio Antonio Carro'; Michael Gabarron \\ Costa $^{1}$ \\ ${ }^{1}$ Universidade do Oeste Paulista - UNOESTE, Presidente Prudente, SP \\ E-mail: brunakushikawa@gmail.com; silvio@unoeste.br; ga_barron@hotmail.com
}

RESUMO - Este trabalho apresenta uma proposta de algoritmo capaz de identificar automaticamente a ocorrência do acidente vascular encefálico (AVC) usando imagens por tomografia computadorizada (TC). São definidos os métodos de segmentação por similaridade e morfologia matemática, além dos filtros de realce utilizados para modificar o histograma da imagem, que compreende os dados de entrada de uma rede neural Perceptron multicamadas, responsável pela classificação. A utilização deste algoritmo para o auxílio ao diagnóstico médico busca agilizar o processo de detecção da doença, de forma precisa e satisfatória, uma vez que a resposta final dada pelo especialista responsável depende de sua subjetividade. $O$ trabalho mostra o desenvolvimento do algoritmo e a análise de seus resultados, que alcança uma acurácia de 98,51\% durante o treinamento de classificação utilizando o filtro de difusão anisotrópica e 91,33\% para segmentação utilizando métodos de limiarização. Uma comparação entre outras técnicas de processamento de imagem e inteligência artificial é realizada, procurando obter a melhor resposta dentro de um modelo novo e de baixo custo.

Palavras-chaves: Acidente Vascular Cerebral, Tomografia Computadorizada, Processamento de Imagens, Redes Neurais.

Recebido em: 30/11/2018

Revisado em: 15/02/2019

Aprovado em: 03/06/2019
ABSTRACT - This work introduce an algorithm proposal able to automatically identify the occurrence of stroke through computed tomography (CT) images. The methods of segmentation by similarity and mathematical morphology are defined, in addition to the enhancement filters used to modify the histogram of the image, which comprises the input data of a Multi-layer Perceptron neural network responsible for classification. The use of this algorithm for medical diagnosis assistance seeks to speed up the process of detection of the disease, accurately and satisfactorily, once the final response given by the responsible specialist depends on his subjectivity. The work comes up with the development of the algorithm and the analysis of its results, which reaches an accuracy of 98,51\% during the classification training using the anisotropic diffusion filter and $91,33 \%$ for segmentation using thresholding methods. A comparison between other image processing 
and artificial intelligence techniques is performed, seeking to obtain the bets response within a new and low cost model.

Keywords: Stroke, Computerized Tomography, Image Processing, Neural Networks

\section{INTRODUÇÃO}

O acidente vascular cerebral (AVC), é a primeira causa de morte e incapacidade no país. É uma doença provocada por uma alteração da irrigação sanguínea, podendo trazer complicações temporárias ou permanentes, que irão depender da intensidade do acidente e de quanto tempo o cérebro ficou sem receber oxigênio apropriadamente. O AVC pode ser dividido em duas categorias principais: o AVC Isquêmico (AVCi), que ocorre devido a uma obstrução arterial, e o AVC Hemorrágico (AVCh), caracterizado por uma ruptura espontânea de um vaso sanguíneo (BRASIL, 2012; CANCELA, 2008; BRAGA; ALVARENGA; MORAES NETO, 2003).

A detecção de AVC em imagens médicas volumétricas é um grande desafio, devido a dificuldade de se diferenciar regiões lesionadas de outras estruturas e tecidos do crânio. Sistemas de Auxílio ao Diagnóstico (computer-aided diagnosis - CAD) utilizam uma grande quantidade de dados extraídos de imagens médicas para auxiliar na localização de regiões suspeitas e classificação de doenças e seus estágios (WANG; SUMMERS, 2012; NUNES, 2006).

Imagens de Tomografia Computadorizada (TC) sem contraste são amplamente utilizadas nos centros de emergência na detecção do AVC, auxiliando especialistas e médicos em seus diagnósticos.

Esse processo depende diretamente da identificação e delineação da região afetada feita visualmente pelo profissional responsável. Como mostra a Figura 1, o AVCi é representado na TC como uma hipodensidade (densidade inferior ao normal), devido à falta de sangue, enquanto que o AVCh é representado por uma hiperdensidade (densidade superior ao normal), devido ao coeficiente de atenuação do sangue ser mais alto do que o do cérebro (FREITAS, 2016; AGUIAR, 2017).

Figura 1. À esquerda encontra-se uma imagem de crânio com $\mathrm{AVCi}$, enquanto à direita encontra-se uma imagem de crânio com AVCh.
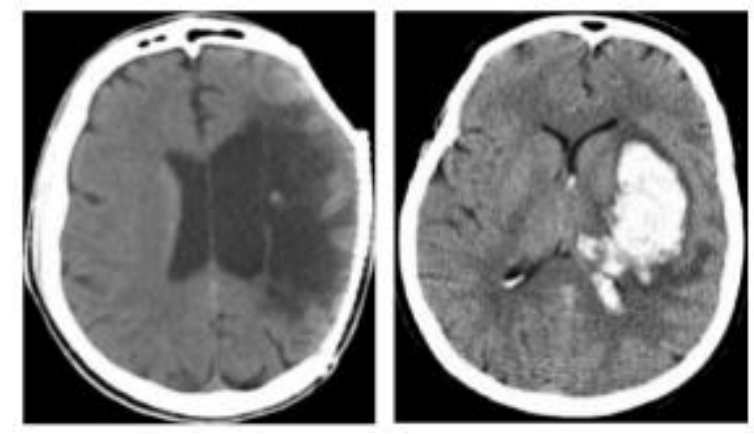

Fonte: (Chawla et al. , 2009).

Este projeto tem como objetivo mostrar o desenvolvimento de um software capaz de identificar o AVC Isquêmico e Hemorrágico, além de segmentar a região encefálica lesionada, através de imagens obtidas por tomografia computadorizada no formato DICOM. Utilizando recursos computacionais relacionados a área da inteligência artificial, buscou-se extrair as informações necessárias com o auxílio de processamento de imagens, e criar uma estrutura de rede neural Perceptron multicamadas, capaz de classificar e segmentar as áreas afetadas pelas duas doenças.

$\mathrm{Na}$ seção 2 são abordados alguns trabalhos que visam a classificação e/ou segmentação de estruturas orgânicas por imagens médicas. Na seção 3 são definidos todos os objetos de estudo envolvidos, desde imagens médicas a técnicas de 
processamento de imagem e inteligência artificial. Na seção 4 está descrito todo o processo de desenvolvimento do algoritmo. $\mathrm{E}$ por fim, as seções 5 e 6 apresentam respectivamente os resultados e discussões sobre todas as técnicas implementadas, enquanto a seção 7 apresenta as últimas considerações sobre o trabalho.

\section{TRABALHOS RELACIONADOS}

Lisowska et al. (2017) propõe uma rede neural convolutiva para detecção de trombos, que é o principal responsável pela ocorrência do AVCi. Trabalha com imagens de tomografia computadorizada sem contraste através da comparação da TC do paciente com um atlas normativo da anatomia encefálica e da análise de simetria. Foi proposta uma rede neural convolucional $3 \mathrm{D}$, denominada Butterfly $C N N$, que possui como entrada dois canais de intensidade que correspondem aos hemisférios direito e esquerdo do cérebro. Chawla et al. (2009) também utiliza o histograma da imagem para realizar a classificação do AVCi e do AVCh

Cavalcante et al. (2013) compara uma rede MLP com o método de Análise de Densidades Pulmonares para detecção de vias aéreas, e exibe os resultados em favor da rede neural, assim como Tarallo (2007), que também mostra como a união de técnicas de processamento de imagem e a MLP são satisfatórias para segmentação e classificação de imagens de úlceras cutâneas.

Freitas (2016) por sua vez, utiliza uma rede neural MLP, cuja função é verificar se o nível de cinza do pixel corresponde ou não a uma região de AVCi. Ele utiliza valores de textura como entrada da rede, calculados através de descritores de Haralick, e, com o auxílio de uma matriz $9 \times 9$, ele analisa a vizinhança de cada pixel para classificar se ele é um ponto da área de um AVCi ou não. Este método, ao contrário do de Aguiar (2017), utiliza a própria rede para segmentar a região de lesionada, enquanto Aguiar apresenta cinco algoritmos baseados em limiarização e em computação natural.

\section{CONCEITOS FUNDAMENTAIS}

\subsection{DICOM}

DICOM

(Digital

Imaging

Communications in Medicine) é uma norma internacional que define os formatos $e$ processos de armazenamento e transmissão de imagem médica digital, possibilitando que imagens e dados associados sejam padronizados para que sejam utilizadas por diferentes equipamentos de imagem, computadores e hospitais (FARIA, 2010).

\subsection{Tomografia Computadorizada}

A TC foi escolhida para este projeto devido a sua majoritária utilização nos centros de emergência, principalmente por seu baixo custo, maior disponibilidade, rapidez e sensibilidade na detecção de tecidos lesionados por AVC, se tornando um dos primeiros exames a serem realizados. Ela se baseia num conjunto de imagens que, quando reagrupadas pelo software de computador, resulta em uma visão muito detalhada e multidimensional dos órgãos internos. Essas imagens possuem informação para discriminar aproximadamente 4000 diferentes níveis (ou tons) de cinza. Esses níveis representam a diferença do coeficiente de atenuação de raios $x$ que um determinado órgão absorve (NUNES, 2006; SAVIONE; GLUECK; MAGALHÃES, 2010).

\subsection{Escala Hounsfield}

A escala utilizada para quantificar corretamente os valores correspondentes aos órgãos, tecidos e estruturas ósseas é a escala de Unidades Hounsfield (HU), que vai de aproximadamente -1024 até 3071. A imagem de um exame de TC não possui valores na escala $\mathrm{HU}$ para fins de armazenamento. Ela deve ser convertida no momento da sua manipulação, utilizando-se a seguinte equação:

$$
\text { hu }=\text { pixel_value } * \text { slope }+ \text { intercept (1) }
$$
, onde hu representa a unidade de saída, pixel_value é o valor armazenado e slope e intercept são tags DICOM que especificam a transformação linear do pixel armazenado 
em disco para sua representação em memória (AGUIAR, 2017).

A escala de Hounsfield permite, através da técnica de janelamento (windowing), a visualização de órgãos específicos que irão se realçar ou atenuar de acordo com os valores aplicados. O janelamento é controlado por dois parâmetros, WW e WL, onde, valores dentro do intervalo definido por $[\mathrm{WL}-W W / 2$, $\mathrm{WL}+\mathrm{WW} / 2$ ] são mapeados em toda a gama de escala de cinza, os tecidos com valores $\mathrm{HU}$ abaixo do intervalo são definidos como preto e os valores acima, como branco (HOUNSFIELD, 1980).

\subsection{Processamento de Imagens}

\subsubsection{Processamento Morfológico}

Erosão: Filtro morfológico que remove da imagem os pixels que não atendem um padrão definido anteriormente. Em geral, reduz as dimensões do objeto, a partir de um elemento estruturante prédefinido. A erosão de $X$ por $B$ é definida como $X \ominus B=\left\{z \mid\left(B_{z}\right) \subseteq X\right.$

, que indica que a erosão de $X$ por $B$ é o conjunto de todos os pontos $Z$ de forma que $B$, transladado por $Z$, está contido em $X$ (GONZALES, WOODS, 2000).

Dilatação: Filtro morfológico que altera uma pequena área representante de um pixel para um dado padrão. Em geral faz com que o objeto cresça no tamanho. A dilatação de $X$ por $B$ é definida como

$X \oplus B=\left\{z \mid(\hat{B})_{z} \cap A \neq \emptyset\right.$

, que indica que a dilatação de $X$ por $B$ é o conjunto de todos os deslocamentos, $Z$, de forma que $\hat{B}$ e $X$ se sobreponham pelo menos por um elemento (GONZALES; WOODS, 2000).

Fechamento: O fechamento é uma operação de dilatação seguida imediatamente de uma erosão utilizando o mesmo elemento estrutural. Ela tende a suavizar contornos, mas geralmente funde partes, elimina pequenos buracos e preenche fendas em um contorno.

\subsubsection{Segmentação}

Limiarização: Método que segmenta imagens em tons de cinza de acordo com uma faixa de intensidade, onde todos os pixels que se encontram dentro dessa faixa são classificados como pertencentes a uma mesma região. Sua representação matemática é dada por:

$g(x, y)=\left\{\begin{array}{l}1, \text { se } i(x, y) \geq T \\ 0, \text { se } i(x, y)<T\end{array}\right.$

onde, $i(x, y)$ representa a imagem de entrada e $g(x, y)$ representa a imagem de saída, binarizada (MILSZTAJN, 2003).

Região: Métodos baseados em regiões abordam a procura de similaridade entre as tonalidades dos pixels ou conjunto de pixels. Uma das técnicas mais conhecidas é a denominada Crescimento de Região, que busca expandir pequenos grupos de pixels a partir de "sementes" (MILSZTAJN, 2003).

\subsubsection{Filtros de Suavização}

Difusão Anisotrópica: Também conhecido como Perona Malik, a difusão anisotrópica é um filtro de suavização que busca manter as bordas da imagem. A ideia é baseada na teoria do espaço-escala, onde a imagem original é encapsulada num conjunto de imagens simplificadas, obtidas pela convolução da imagem original com um filtro Gaussiano $G(x, z ; t)$, com variância definida por um parâmetro de escala $t$ (MIRANDA; CAMARGO NETO, 2006). Esta convolução é definida por

$G(x, y ; t)=\frac{1}{2 \pi t} e^{-\left(x^{2}+y^{2}\right) / 2 t}$

Kuwahara: O filtro de Kuwahara é umas das primeiras técnicas de suavização de borda. Ele utiliza uma máscara de tamanho $4 * n-1$, possibilitando que a janela da imagem possa ser dividida em quatro quadrados menores. O pixel central da janela é então substituído pela média da subjanela com menor valor de variância (SANCHES et al., 2015).

Gaussiano: A suavização da imagem é realizada por meio da substituição de cada pixel por uma média ponderada dos pixels vizinhos, tal que o peso dado a um vizinho decresce monotonicamente com a distância do pixel central. 
A largura de um filtro Gaussiano, ou seja, seu grau de suavização está relacionado com o parâmetro $\sigma$. Quanto maior o valor de $\sigma$, maior a largura do filtro Gaussiano e maior o seu grau de suavização (SANCHES et al., 2015).

\subsubsection{Histograma}

O histograma fornece a informação de quantos pixels possuem um determinado nível de cinza, permitindo que possamos observar níveis de contraste e de tons médios (claro e escuro) de uma imagem a partir de grandezas numéricas (GONZALES; WOODS, 2000). Ele é definido por

$h(i)=\sum_{m=0}^{M-1} \quad \sum_{n=0}^{N-1} \quad \delta(f(m, n)-i), i=$
$0,1 \ldots, 255 .(6)$
onde,

$$
\delta(w)=\left\{\begin{array}{l}
1, w=0 \\
0, \text { outros casos }
\end{array} .\right.
$$

\subsection{Redes Neurais Artificiais}

Redes Neurais Artificiais (RNA) são modelos computacionais baseados em redes neurais biológicas. É utilizada no reconhecimento e classificação de padrões através de elementos de processamento altamente conectados capazes de fazer computações elementares (WANG; SUMMERS, 2012).

$\mathrm{Na}$ área de processamento de imagens, as RNAs são utilizadas devida a sua capacidade de extrair poder computacional através de paralelismo, generalização, autoorganização, tolerância a falhas e aprendizagem através de exemplos.

Ela utiliza uma unidade independente de processamento de informações, o neurônio, que, quando conectado a outros neurônios, formam uma complexa estrutura de circuito cerebral. Em uma RNA, o neurônio é representado por um elemento processador que possui diversas entradas $(x)$, cada uma com um peso sináptico (w). Esses pesos são processados pela função de soma $\left(\sum\right)$ e passam por uma função de ativação $(g()$.$) , que irá gerar a saída do$ elemento processador (LIBERMAN, 1997).
Figura 2. Modelo matemático de um neurônio.

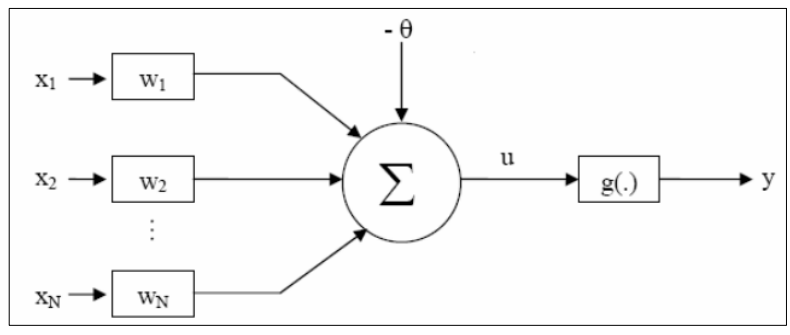

Fonte: (Tarallo, 2007).

A MLP é um dos métodos mais adotados devido a sua capacidade de adaptação a qualquer tipo de padrão. Ela utiliza a aprendizagem supervisionada, onde a entrada e a saída desejada da rede são fornecidas por um supervisor (professor) externo.

Figura 3. Arquitetura de uma rede MLP.

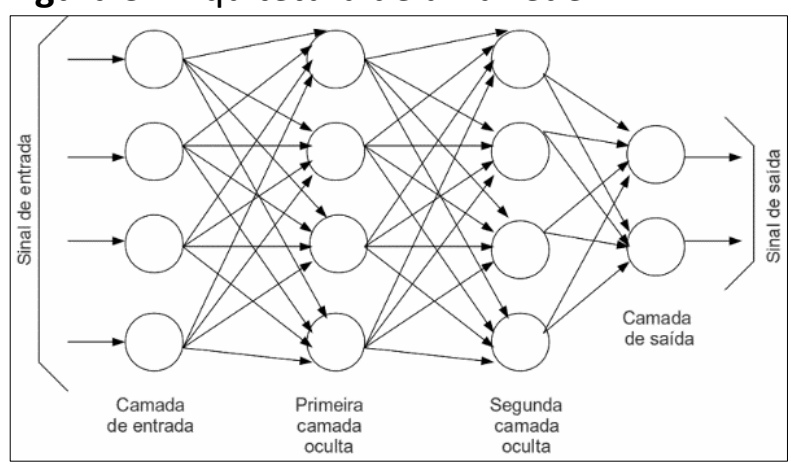

Fonte: (monolitonimbus.com.br).

A camada de entrada é onde os padrões são apresentados, enquanto as ocultas são responsáveis pela extração de características, e por fim a camada de saída, que dá a resposta da rede para as entradas apresentadas.

O objetivo é ajustar os parâmetros da rede de forma a encontrar uma ligação entre os pares de entrada e saída fornecidos. Esse ajuste é feito pelo algoritmo de retropropagação, executado até que o erro médio da rede seja suficientemente pequeno ou até atingir um número determinado de iterações. O aprendizado ocorre quando a rede atinge uma solução generalizada para uma classe de problemas. 


\section{METODOLOGIA}

\subsection{População de Amostra}

Para o desenvolvimento do software foram incluídos pacientes que realizaram um exame de tomografia computadorizada de crânio no Hospital Regional de Presidente Prudente, no período de 01/11/2018 a 10/12/2018 sob aprovação do Comitê de Ética da UNOESTE - Universidade do Oeste Paulista, CAAE no 99393318.0.0000.5515.

Os exames de nove pacientes foram utilizados, sem reformatação, rotulados em "isquemia", "hemorragia" e "saudável". Ao total, 450 imagens foram utilizadas para todo o processo.

\subsection{Protocolo de Desenvolvimento}

A ferramenta proposta foi desenvolvida em duas linguagens, sendo uma delas o Java, utilizado para composição da interface devido a sua ampla variedade de ferramentas voltadas a interação com o usuário, que faz uma ponte com a segunda linguagem, o Python, voltado principalmente para aplicações que demandam muito processamento.

O processo de estudo foi dividido em três etapas, sendo a primeira o préprocessamento da imagem e a extração das informações relevantes para as redes, a segunda, estudo dos resultados de cada rede para cada tipo de imagem pré-processada, para análise da melhor combinação para classificação, e a terceira etapa, segmentação da lesão.

A Figura 4 apresenta um fluxograma com todos os passos, desde a aquisição e tratamento $\mathrm{da}$ imagem até a sua segmentação, indicando a ordem em que os métodos foram utilizados.
Figura 4. Fluxograma do algoritmo desenvolvido.

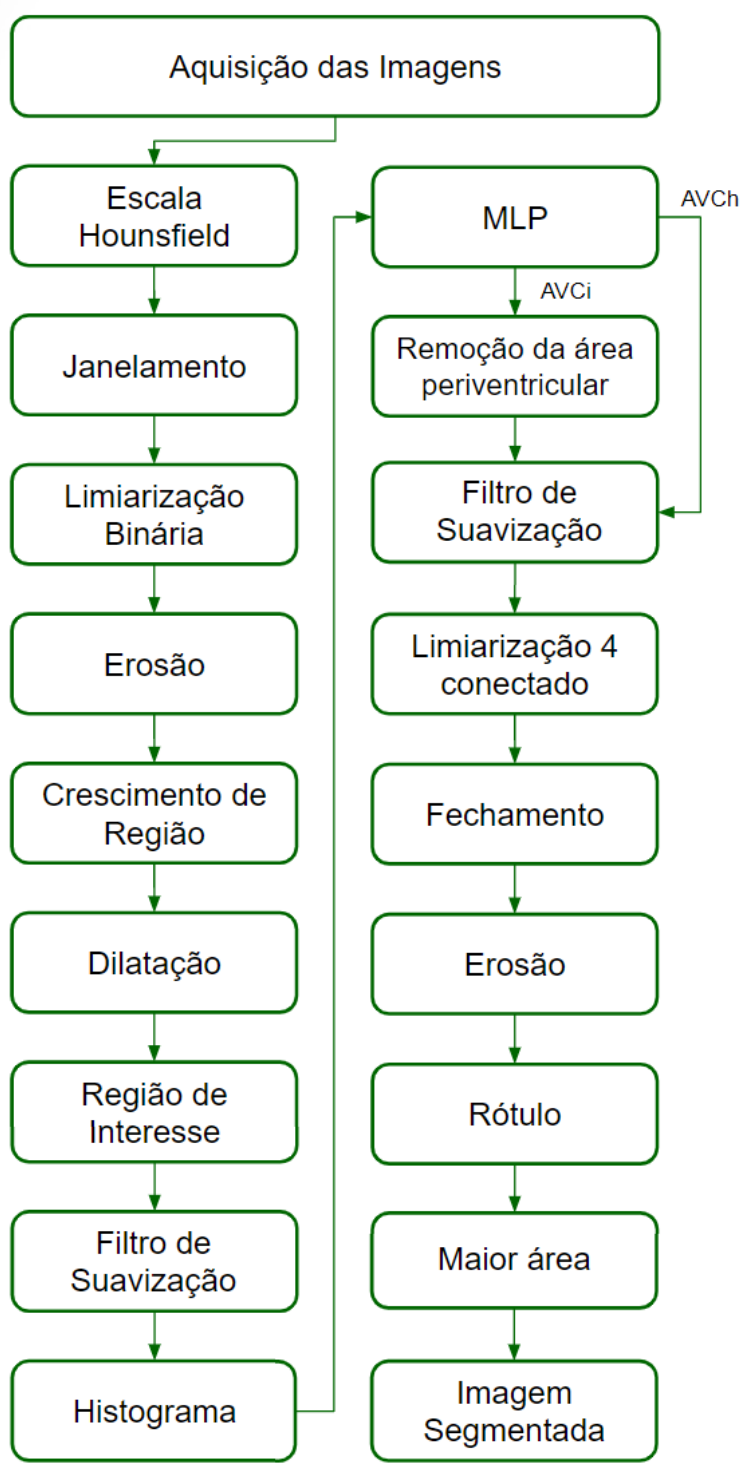

Fonte: $\mathrm{O}$ autor.

A manipulação das imagens no formato DICOM foi possível através da biblioteca PyDicom, que permite a leitura dos cabeçalhos do exame, além da disponibilização da imagem em tons de cinza no formato de um numpy array de duas dimensões.

Inicialmente foram analisadas quais fatias da tomografia retratam melhor a presença da doença. $O$ intervalo escolhido inclui as fatias de 40 a 90 , totalizando 50 imagens por exame. As fatias excluídas compreendem imagens cujo padrão de processamento escolhido inviabiliza a sua análise. 
A manipulação das imagens se iniciou aplicando a equação linear da escala Hounsfield sob os dados puros da TC. Em seguida, foi realizado o janelamento com os valores de $W W=80$ e $W C=40$, valores indicados para visualização de tecidos do cérebro. A Figura 5 mostra a diferença entre a imagem pura provida no exame (Figura 5a), e a mesma imagem após aplicação da escala e do janelamento (Figura $5 b$ ).

Figura 5. Aplicação da escala Hounsfield e do janelamento.

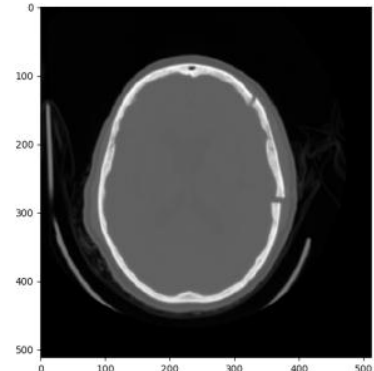

(a)

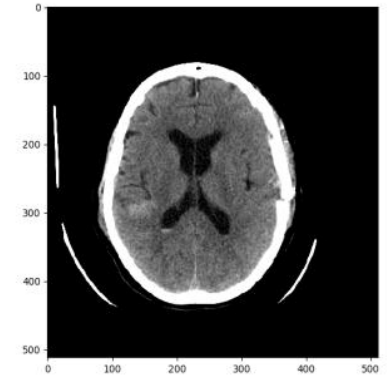

(b)
Fonte: $\mathrm{O}$ autor.

A extração da região de interesse começa por uma limiarização de limiares arbitrários, de valores -300 e 150, definido por Aguiar (2017) em seu trabalho, para exclusão dos ossos e do ar (Figura 6a)

Foi então aplicada uma erosão de máscara $10 \times 10$, utilizando a biblioteca scikitimage, para a exclusão de áreas indesejadas (Figura 6b), seguida de uma segmentação de região interna do cérebro, utilizando o algoritmo Flood Fill do OpenCV, com um valor de semente arbitrário de 256,325 (Figura 6c). Para retomar as dimensões de interesse que foram reduzidas durante a erosão, uma dilatação de mesma máscara foi aplicada, permitindo a obtenção da imagem binarizada que define a máscara da região de interesse (Figura 6d).
Figura 6. Imagens obtidas durante a etapa de segmentação da região de interesse.

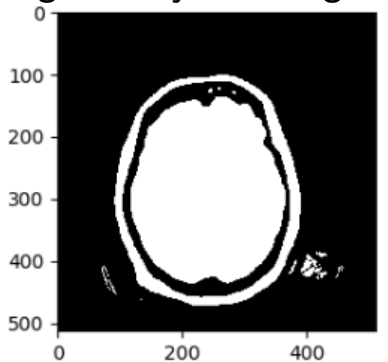

(a)

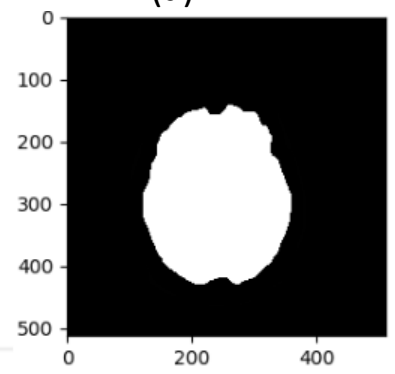

(c)

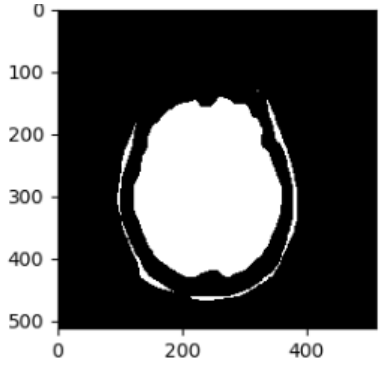

(b)

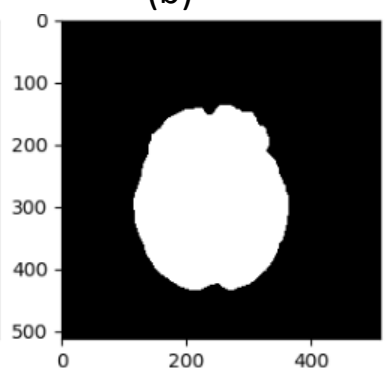

(d)
Fonte: O autor

A região de interesse pôde ser então extraída após a multiplicação da região extraída nos passos descritos anteriormente com a imagem obtida no janelamento (Figura 5b). O resultado desta etapa pode ser visto na Figura 7.

Figura 7. Região de Interesse.

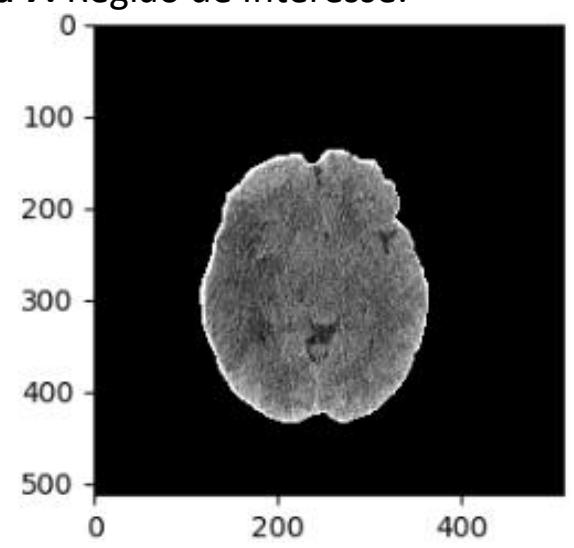

Fonte: O autor

Visando melhorar a performance da classificação, dois algoritmos de suavização foram estudados e comparados, analisandose o resultado final da rede MLP quando aplicados ambos os filtros. O primeiro se baseia no filtro de suavização de difusão anisotrópica, aplicada com o auxílio da biblioteca MedPy. O segundo, baseado no filtro de Kuwahara, foi implementado em 
cima de um código aberto da biblioteca Matlab. Dessa região de interesse foi extraído o histograma, com 79 valores referentes aos 80 tons de cinza janelamento, com excessão do valor correspondente ao tom 0 , devido a sua disparidade dos outros.

O histograma, juntamente com a idade e o sexo do paciente extraídos do cabeçalho da imagem DICOM, foi utilizado como entrada de uma rede MLP, implementada com o auxílio da biblioteca Keras, própria para trabalhos com redes neurais.

A rede possui uma camada de entrada com 81 neurônios, duas camadas ocultas, com 128 e 100 neurônios respectivamente, porém ambas com a mesma função de ativação Relu. A camada de saída possui três neurônios, que identificam os três status desejados, isquêmico, hemorrágico ou saudável, e a sua função de ativação é do tipo softmax.

Entre as camadas foi adicionado uma camada de Dropout, responsável por ativar ou não uma determinada porcentagem de neurônios, para que se evite o overfitting, e a rede consiga generalizar sua classificação.

A última etapa do trabalho, a segmentação, foi realizada baseando-se na resposta da rede. No caso de crânios classificados como saudáveis, nenhum processamento é feito. No caso dos isquêmicos, uma substituição dos pixels da região onde são localizados os ventríloquos foi feita, para que não se tenha problema com a segmentação baseada em limiarização, uma vez que, dependendo da intensidade da lesão, suas escalas na unidade Hounsfield podem ser muito próximas. A representação desta etapa pode ser vista na Figura 8.
Figura 8. Imagem do retângulo contendo o ventrículo e a área periventricular.

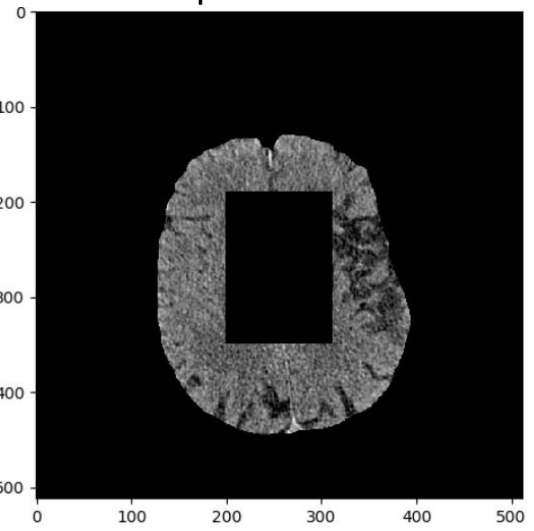

Fonte: $\mathrm{O}$ autor.

A segmentação da região lesionada através de métodos de threshold é feita sob a imagem obtida na etapa de extração da região de interesse (Figura 7) e inicia com um filtro de suavização gaussiano de sigma igual a 1.5 (Figura 9a). Em seguida um threshold 4 conectado é aplicado, com um intervalo pré definido para cada subtipo do AVC, sendo para o AVC isquêmico o intervalo de 5 a 20, e para o AVC hemorrágico, de 45 a 70. Caso o pixel central esteja dentro do intervalo, uma análise de seus quatro vizinhos é feita, e, em caso de todos estarem dentro deste mesmo intervalo, todos são considerados dentro da região de interesse da segmentação (Figura 9b).

Então é aplicado um fechamento cujo elemento estruturante é um plano em forma de disco, de raio 4 (Figura 9c), seguido de uma erosão de máscara 4x4 (Figura 9d).

Figura 9. Imagens obtidas durante a etapa de segmentação da lesão.

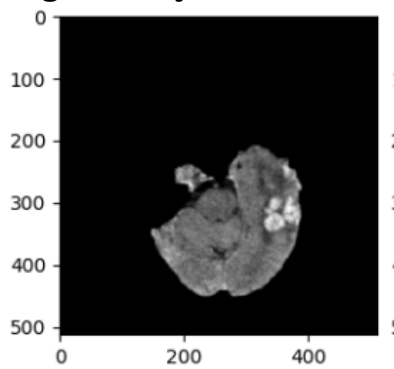

(a)

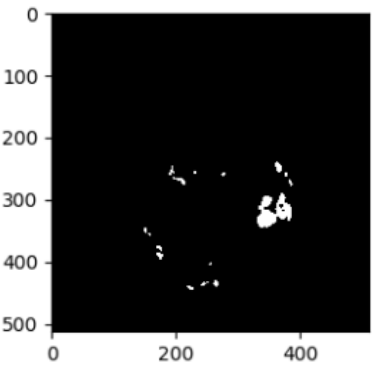

(b) 


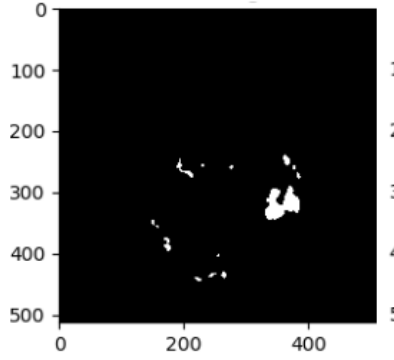

(c)

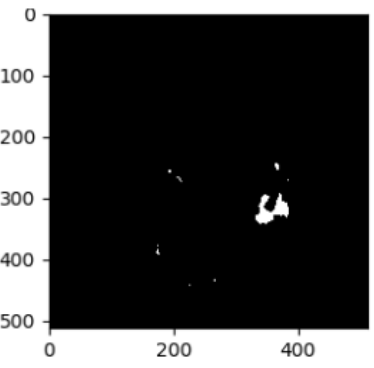

(d)
Fonte: $\mathrm{O}$ autor.

Depois de rotuladas, a região de maior área é selecionada com a que melhor representa a lesão. Ela é aplicada sob a imagem obtida no processo de janelamento (Figura 5b) de forma a ressaltar a região como mostra a Figura 10.

Figura 10. Resultado do processo de segmentação por limiarização.

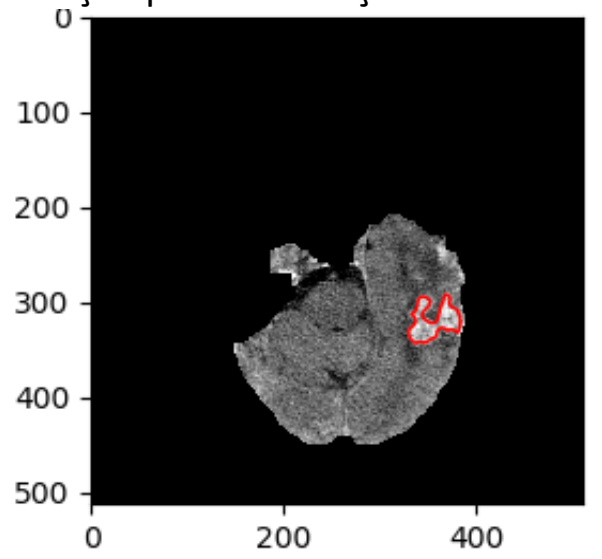

Fonte: $\mathrm{O}$ autor.

Para completar a análise do resultado do modelo, mais dois algoritmos foram implementados, uma rede neural convolucional (CNN) para comparação entre as classificações e um algoritmo de clusterização por tom de cinza para comparação dos métodos de segmentação.

Os dados de entrada da CNN correspondem as imagens obtidas no processo de janelamento. Depois as imagens passam por duas camadas de convolução (extraem características usando quadrados menores da imagem de entrada), cada uma seguida por uma camada de pooling (reduzem as dimensões da imagem mas retém as informações importantes) e uma camada de Dropout. Em seguida os dados são vetorizados pela camada de Flatten e entram em uma rede totalmente conectada com três neurônios de saída onde ocorre a classificação.

O K-means por sua vez, é um algoritmo de clusterização que visa particionar os dados em um número de $k$ grupos, tendo em vista o grupo que está mais próximo da média. Em imagens, ele pode ser utilizado para segmentar regiões de acordo com a cor, possibilitando a visualização de $k$ tons da imagem, que é útil para se extrair regiões que se adequam a um problema específico.

\section{RESULTADOS}

Os resultados obtidos a partir do treinamento da rede MLP com um dataset de treino de 315 imagens e 135 para testes são descritos na Tabela I. Há também nesta mesma tabela, uma comparação dos resultados obtidos pelo método proposto e rede neural convolucional (CNN).

Tabela 1. Acurácia do treinamento da rede MLP.

\begin{tabular}{|c|c|c|}
\hline $\begin{array}{c}\text { MLP + } \\
\text { Difusão } \\
\text { Anisotrópica }\end{array}$ & $\begin{array}{c}\text { MLP + } \\
\text { Kuwahara }\end{array}$ & CNN \\
\hline $98,51 \%$ & $95,55 \%$ & $96,29 \%$ \\
\hline
\end{tabular}

Fonte: $\mathrm{O}$ autor.

A CNN foi escolhida como método de comparação devido a sua vasta utilização em problemas de classificação que tem como objeto de estudo imagens digitais, principalmente em aplicações para o ramo da medicina.

Para a segmentação, quatro métodos foram comparados e avaliados, dois métodos utilizando limiarização, e mais dois utilizando $o$ algoritmo de K-means.

A diferença entre 0 método de limiarização 1 e 2, é que o 1 utiliza uma limiarização que leva em conta os 4 vizinhos mais próximos de cada pixel, enquanto que o 2 apenas aplica a limiarização para toda a 
imagem, independentemente da análise da região ao redor.

Os resultados obtidos foram os descritos pelas Tabelas II e III, que representam a eficácia dos métodos na segmentação de isquemia e hemorragia respectivamente, baseados em uma matriz de confusão.

Tabela 2. Segmentação para isquemia.

\begin{tabular}{|c|c|c|c|c|}
\cline { 2 - 5 } \multicolumn{1}{c|}{} & Limiar 1 & Limiar 2 & $\mathrm{k}=5$ & $\mathrm{k}=6$ \\
\hline $\begin{array}{c}\text { Verdadeiro } \\
\text { Positivo }\end{array}$ & 112 & 113 & 83 & 71 \\
\hline $\begin{array}{c}\text { Verdadeiro } \\
\text { Negativo }\end{array}$ & 11 & 4 & 13 & 14 \\
\hline $\begin{array}{c}\text { Falso } \\
\text { Positivo }\end{array}$ & 11 & 24 & 16 & 13 \\
\hline $\begin{array}{c}\text { Falso } \\
\text { Negativo }\end{array}$ & 16 & 12 & 38 & 52 \\
\hline Acurácia & 0,82 & 0,78 & 0,64 & 0,566 \\
6
\end{tabular}

Fonte: $\mathrm{O}$ autor.

Tabela 3. Segmentação para hemorragia.

\begin{tabular}{|c|c|c|c|c|}
\hline & & & & \\
\hline & Limiar 1 & Limiar 2 & $k=5$ & $k=6$ \\
\hline $\begin{array}{c}\text { Verdadeiro } \\
\text { Positivo }\end{array}$ & 95 & 95 & 68 & 70 \\
\hline $\begin{array}{c}\text { Verdadeiro } \\
\text { Negativo }\end{array}$ & 42 & 42 & 42 & 41 \\
\hline $\begin{array}{l}\text { Falso } \\
\text { Positivo }\end{array}$ & 3 & 3 & 5 & 7 \\
\hline $\begin{array}{c}\text { Falso } \\
\text { Negativo }\end{array}$ & 10 & 10 & 35 & 32 \\
\hline Acurácia & 0,9133 & 0,9133 & $\begin{array}{c}0,733 \\
3\end{array}$ & 0,74 \\
\hline
\end{tabular}

Fonte: $\mathrm{O}$ autor.

\section{DISCUSSÕES}

O filtro de suavização de difusão anisotrópica se mostrou superior ao filtro de Kuwahara para a classificação realizada pela Rede Neural Perceptron Multicamadas, cuja entrada era composta pelo histograma da região de interesse da imagem após aplicação do filtro.

Em comparação com os resultados obtidos pela rede CNN, cuja entrada era formada por imagens processadas até a fase do janelamento, a MLP se mostrou mais adequada. Uma das condições para a resposta da CNN não alcançar os valores de acurácia desejados é devido a falta de generalização dos exames.

O modelo de segmentação proposto, baseado em threshold apresentou melhores resultados em comparação com o K-means, destacando-se o método de limiarização 1 , que mesmo trazendo um valor de acurácia igual ao método de limiarização para a segmentação de regiões de AVC hemorrágico, é superior ao mesmo na segmentação de regiões de AVC isquêmico.

\section{CONSIDERAÇÕES FINAIS}

O levantamento de dados foi uma difícil etapa durante a realização deste trabalho devido principalmente ao reduzido número de exames disponibilizados dentro do tempo proposto. Em função disto, recomenda-se para trabalhos futuros a incorporação de novos exames que permitam não apenas melhorar a resposta de classificação, mas também minudenciar os subtipos de lesões isquêmicas e hemorrágicas.

Sugere-se também a implementação de uma função que compare as intensidades de cinza dos dois hemisférios do cérebro antes da etapa de limiarização, de forma a eliminar regiões simétricas que poderiam ser descartadas pelo threshold na fase de segmentação das lesões.

Por fim, este trabalho mostrou como sistemas computacionais possuem inúmeras 
possibilidades de aplicação na área da medicina, buscando complementar e auxiliar profissionais de todas as especialidades.

Ainda é um desafio ver esses sistemas sendo utilizados na prática, e muitas coisas ainda podem ser melhoradas e até descobertas. Porém, é necessário que haja apoio e incentivo a essa interdisciplinaridade, para que o avanço possa acontecer de maneira benéfica e próspera.

\section{REFERÊNCIAS}

AGUIAR, Cecília Burle de. Avaliação de acidente vascular cerebral em tomografia computadorizada utilizando algoritmo de otimização de formigas. 2017. Dissertação (Mestrado em Engenharia Elétrica) - Instituto Federal de Educação, Ciência e Tecnologia da Paraíba - IFPB.João Pessoa - PB, 2017.

BRAGA, Jorge Luiz; ALVARENGA, Regina M. P.; MORAES NETO, João. B. Mascarenha de. Acidente vascular cerebral. Rev Bras Med, v. 60, n. 3, p. 88-94, 2003.

BRASIL. Ministério da Saúde. Acidente vascular cerebral (AVC). Brasília, 2017. Disponível em:

http://www.brasil.gov.br/noticias/saude/201 2/04/acidente-vascular-cerebral-avc. Acesso em: 17 ez. 2018.

CANCELA, Diana Manuela Gomes. $O$ acidente vascular cerebral-classificação, principais consequências e reabilitação. 0 portal do Psicólogo, Portugal, p. 2-18, 2008.

CAVALCANTE, Tarique da Silveira et al. Segmentação automática 2D de vias aéreas em imagens de tomografia computadorizada do tórax. Revista Brasileira de Engenharia Biomédica, [s.l.], v. 29, n. 4, p.389-403, 2013.. http://dx.doi.org/10.4322/rbeb.2013.038.

CHAWLA, Mayank et al. A method for automatic detection and classification of stroke from brain CT images. Annual International Conference of the IEEE
Engineering In Medicine And Biology Society. set. 2009. http://dx.doi.org/10.1109/iembs.2009.53352 89.

FARIA, Diego. Análise e Processamento de Imagem. Faculdade de Engenharia da Universidade do Porto. Jun 2010.

FREITAS, Emannuel D. G. de. Segmentação de Regiões de AVC Isquêmicos em Imagens de TC por meio da Classificação de Textura. 2016. Dissertação (Mestrado em Engenharia Elétrica) - Instituto Federal de Educação, Ciência e Tecnologia da Paraíba - IFPBJoão Pessoa, PB, 2016. http://dx.doi.org/10.21528/CBIC2015-062.

HOUNSFIELD, G. N. Computed Medical Imaging. Journal of Computer Assisted Tomography, v. 4, n. 5, p. 665-674, 1980. https://doi.org/10.1097/00004728198010000-00017

LIBERMAN, Felipe. Classificação de imagens digitais por textura usando redes neurais. 1997. Dissertação (Mestrado em Ciência da Computação) - Instituto de Informática, Universidade Federal do Rio Grande do Sul. Porto Alegre, 1997.

LISOWSKA, Aneta et al. Thrombus Detection in CT Brain Scans using a Convolutional Neural Network. Proceedings of the 10th International Joint Conference On Biomedical Engineering Systems And Technologies, 2017. Science and Technology Publications. http://dx.doi.org/10.5220/000611460024003 $\underline{3}$.

MILSZTAJN, Flávio. Segmentação de tecidos cerebrais em imagens de ressonância magnética utilizando campos aleatórios de Markov. 2003. Dissertação (Mestrado em Informática) -Universidade Federal do Paraná, Curitiba, 2003.

MIRANDA, José I.;CAMARGO NETO, João.Modelo de difusão anisotrópica para 
detecção de bordas. Embrapa Informática Agropecuária. Comunicado técnico, Campinas, v.72, nov.2007.

NUNES, Fátima L. S. Atualizações em Informática: introdução ao processamento de imagens médicas para auxílio ao diagnóstico - uma visão prática. Rio de Janeiro: Ed.PUC-Rio: SBC, 2006. Cap. 2.

SANCHES, Carlos $H$. et al. Técnicas de Suavização de Imagens e Eliminação de Ruídos. In: EATI - ENCONTRO ANUAL DE TECNOLOGIA DA INFORMAÇÃO.. 6., 2015. Frederico Westphalen.Anais [...].Frederico Westphale: Instituto Federal Farroupilha,2015.

SAVIONE, Herick; GLUECK, Flávio; MAGALHÃES, Fabíola C. R.. Tomografia
Computadorizada. 3. ed. Belo Horizonte: Faculdade Novo Rumo,, 2010.

TARALLO, André S.. Segmentação e Classificação de Imagens Digitais de Úlceras Cutâneas através de Redes Neurais Artificiais. 2007. Dissertação (Mestrado em Engenharia Elétrica) - Departamento de Engenharia Elétrica, Escola de Engenharia de São Carlos - Universidade de São Paulo, São Carlos - SP, 2007.

WANG, Shijun; SUMMERS, Ronald M. Machine Learning and Radiology. Med Image Analv. 16, n. 5, p. 933-951, 2012. https://doi.org/10.1016/i.media.2012.02.005 